\title{
Announcement: Howard Rosenbrock Prize 2019
}

\author{
Nikolaos V. Sahinidis ${ }^{1}$
}

Received: 30 June 2020 / Revised: 30 June 2020 / Accepted: 30 June 2020 / Published online: 10 July 2020 (c) Springer Science+Business Media, LLC, part of Springer Nature 2020

I am pleased to announce the winners of the 2019 Rosenbrock Prize. This prize is awarded annually to honor the authors of the best paper published in Optimization and Engineering (OPTE) in the previous year. The award includes a $\$ 500$ prize sponsored by Springer. Past recipients of the prize were Jason E. Hicken in 2014 (Hicken 2014), Moritz Simon and Michael Ulbrich in 2015 (Simon and Ulbrich 2015), Taedong Kim and Stephen J. Wright in 2016 (Kim and Wright 2016), Hoai An Le Thi and Tao Pham Dinh and 2017 (Le Thi and Dinh 2017), and Laurent Hoeltgen, Michael Breuß, Gert Herold, and Ennes Sarrad in 2018 (Hoeltgen et al. 2018).

The winners of the 2019 Rosenbrock Prize are Robert Burlacu, Herbert Egger, Martin Groß, Alexander Martin, Marc E. Pfetsch, Lars Schewe, Mathias Sirvent, and Martin Skutella for their work titled Maximizing the storage capacity of gas networks: a global MINLP approach (Burlacu et al. 2019). Robert Burlacu, Alexander Martin, Lars Schewe and Mathias Sirvent are at Friedrich-Alexander-Universität Erlangen-Nürnberg, Herbert Egger and Marc E. Pfetsch are at Technische Universität Darmstadt, Martin Groß is at RWTH Aachen, and Martin Skutella is at Technische Universität Berlin.

This year's winning paper was selected by a committee composed of the committee-chair Marina Epelman (University of Michigan, USA) and committee-members Michel Gamache (Polytechnique Montreal, Canada) and Luis Zuluaga (Lehigh University, USA). The group produced the following citation for the winning paper:

This paper considers the problem of maximizing the storage capacity of gas networks - a problem of increasing importance in the operation of energy networks, especially in the context of interrelated electric and gas networks. The proposed Mixed Integer Nonlinear Programming formulation incorporates a transient model of nonlinear gas physics derived from the Euler equations, and the discrete switching of active elements such as valves and compressors. It is based on a new discretization approach over space and time, whose wellposedness is rigorously established in the paper, and solved using an algorithm

Nikolaos V. Sahinidis

sahinidis@cmu.edu

1 Department of Chemical Engineering, Carnegie Mellon University, Pittsburgh, PA, USA 
based on adaptive MIP relaxations of the MINLP. The algorithm is refined by using problem-specific improvements of primal and dual bounds. This paper is a great example of research into an important and complex engineering problem through new optimization modeling approaches and extensions of sophisticated solution algorithms.

The Rosenbrock Prize recognizes excellent research contributions that exemplify the commitment to bridge the gap between optimization and engineering that characterized the original work of the award's namesake, Howard Rosenbrock. OPTE provides a vibrant intellectual forum for researchers in engineering and optimization. We publish new developments in optimization as well as challenging and successful applications of optimization in engineering. Researchers from around the world are encouraged to submit high-quality papers exploring algorithms, software, and theory at the interface of optimization and engineering.

\section{References}

Burlacu R, Egger H, Groß M, Martin A, Pfetsch ME, Schewe L, Sirvent M, Skutella M (2019) Maximizing the storage capacity of gas networks: a global MINLP approach. Optim Eng 20:543-573

Hicken JE (2014) Inexact Hessian-vector products in reduced-space differential-equation constrained optimization. Optim Eng 15:575-608

Hoeltgen L, Breuß M, Herold G, Sarradj E (2018) Sparse $\ell_{1}$ regularisation of matrix valued models for acoustic source characterisation. Optim Eng 19:39-70

Kim T, Wright SJ (2016) An S $\ell_{1}$ LP-active set approach for feasibility restoration in power systems. Optim Eng 17:385-419

Le Thi H A, Dinh T Pham (2017) Difference of convex functions algorithms (DCA) for image restoration via a Markov random field model. Optim Eng 18:873-906

Simon M, Ulbrich M (2015) Adjoint based optimal control of partially miscible two-phase flow in porous media with applications to $\mathrm{CO}_{2}$ sequestration in underground reservoirs. Optim Eng 16:103-130

Publisher's Note Springer Nature remains neutral with regard to jurisdictional claims in published maps and institutional affiliations. 